\title{
Vaccine mandates, public trust, and vaccine confidence: understanding perceptions is important
}

\author{
Roy Widdus ${ }^{1} \cdot$ Heidi Larson ${ }^{2}$
}

Published online: 22 March 2018

(C) The Author(s) 2018. This article is an open access publication

\begin{abstract}
The experience in Australia with penalizing parents who refuse to have their children vaccinated demonstrates the need to study and understand resistance to vaccination as a global phenomenon with particular local manifestations.
\end{abstract}

Keywords Vaccines - Penalties for vaccine resistance Societal benefits - Vaccine efficacy

In this issue, Helps, Leask, and Barclay [1] investigate the impacts of Australia's "no jab, no pay, no play" legislation that withholds daycare benefits from those who do not get their children vaccinated. Their findings reveal the potential unintended consequences of such legislation and consider the implications for interventions as well as policies.

A previous exhortation [2] for more efforts to understand the reasons for resistance to vaccination was prompted by a study in Pakistan that documented the opinions in local populations that complicated the end-game for polio eradication [3]. Both studies show the complexity and diversity of reasons why vaccination is not universally perceived by all decision making parents as a 'public good.'

Vaccination programs and policies to promote vaccination can falter or fail because they do not fully understand the perceptions, values, and beliefs that drive vaccination choices. Negative beliefs with respect to vaccination may be grounded in differences of opinion about the technical efficacy or safety of vaccines, but they

Roy Widdus

roy.widdus@icloud.com

Heidi Larson

Heidi.Larson@LSHTM.ac.uk

1 Global Health Futures, 129 Wappoo Landing Circle, Charleston, SC 29412, USA

2 Vaccine Confidence Project, London School of Hygiene and Tropical Medicine, London, UK 
can go beyond the strictly technical. They can be religious, philosophical, or even 'political,' if one considers public opposition to government intrusion into health decisions. Sometimes beliefs about vaccination may also be based on misinformation or unfounded suspicion of intentional harm-sterilization, for exampleespecially in populations that are disadvantaged. These beliefs are nonetheless the basis for individuals' decisions. Only by understanding the origins and propagation of all these types of belief, and how they 'mutate' to retain credibility amongst vaccination resisters, will proponents of vaccination be able to alter such beliefsand even then vaccine acceptance is not guaranteed. The laudable Vaccine Confidence Project (www.vaccineconfidence.org) tracks the global picture of trends and issues in vaccine confidence, but given the locally diverse nature of the issues, more studies are needed at a regional or very local level.

Lower than optimal vaccination rates pose the threat of outbreaks of previously controlled infectious diseases. Where the vaccination resisters are locally clustered, these outbreaks will come sooner and the toll will be proportionally larger because transmission will be more efficient. Those who cannot be vaccinated for medical reasons and those who are vaccinated but have a low immunological response are put at extra risk of disease because of decisions by others to not vaccinate.

Efforts to combat resistance to vaccination usually communicate information on vaccine safety, or are, in some measure, 'coercive' nudges. Widely supported measures in the latter category can include instituting requirements for vaccination for entry into daycare, school, or college. There has been a trend in recent years to eliminate religious or philosophical exemptions from required vaccination. More forceful_-some would say punitive-approaches include those such as introduced in Australia, where societal benefits were not paid to those who have resisted vaccination. In some countries, there is 'folk memory'-possibly true-of physically forced vaccination against smallpox. This demonstrates how even good intentions can have negative outcomes, if not attentive to implications for public trust.

Responses to such vaccination promotion efforts are sometimes promising, but often the law of unintended consequences prevails. In Australia, the "no jab, no pay, no play" was intended to increase compliance with vaccination, but instead "It just forces hardship" as Helps and her co-authors describe, leading to children not benefitting from daycare, parents reducing work and study to take care of children, and in an even firmer commitment to not vaccinating - at least among those who were initially resistant, the primary target of the legislation.

Overall vaccination coverage around the world has not increased significantly in the last few years, although vaccines to protect against additional diseases are reaching those who do get vaccinated. Part of the explanation for stalled coverage is likely to be widespread questioning of vaccination and distrust in government authorities.

The two articles that stimulated this and the previous commentary had different foci, but highlighted a common concern. All countries should recognize that public confidence in vaccination is more easily undermined than currently anticipated. It is time for vaccination programs to recognize that in this era when increasingly facts alone do not drive decisions, it is important to understand the complex and diverse 
perceptions held by vaccination resisters. To help design effective interventions, there must be equal attention to, and investment in, understanding of what shapes the beliefs and decisions of those who accept vaccination and those segments of the population who are hesitant.

Open Access This article is distributed under the terms of the Creative Commons Attribution 4.0 International License (http://creativecommons.org/licenses/by/4.0/), which permits unrestricted use, distribution, and reproduction in any medium, provided you give appropriate credit to the original author(s) and the source, provide a link to the Creative Commons license, and indicate if changes were made.

\section{References}

1. Helps C, Leask J, Barclay L. "It just forces hardship": the impact of government financial penalties on non-vaccinating parents. J Public Health Policy. 2018. https://doi.org/10.1057/s41271-017-0116-6.

2. Habib MA, et al. Knowledge and perceptions of polio and polio immunization in polio high-risk areas of Pakistan. J Public Health Policy. 2017;38(1):16-36.

3. Widdus R. Commentary: understanding 'perceptions' is critical for all vaccination efforts, not only polio eradication. J Public Health Policy. 2017;38(1):37-8.

Roy Widdus $\mathrm{PhD}$ is a Microbial Biochemist with training in epidemiology. He worked on vaccine issues at the US National Academy of Sciences Institute of Medicine. He was the Coordinator, of the Children's' Vaccine Initiative based in Geneva, Switzerland.

Heidi Larson PhD directs the Vaccine Confidence Project at the London School of Hygiene and Tropical Medicine. 